\title{
The usefulness of trabecular bone score in patients with ankylosing spondylitis
}

\author{
Seoung Wan Nam ${ }^{1}$, Yoon-Kyoung Sung ${ }^{2}$, Dam Kim³ ${ }^{3}$, Soo-Kyung Cho ${ }^{2}$, Yoonah Song ${ }^{4}$, Yun Young Choi ${ }^{5}$, \\ Yongjin $\mathrm{Sim}^{6}$, and Tae-Hwan $\mathrm{Kim}^{2}$
}

\begin{abstract}
${ }^{1}$ Department of Rheumatology, Wonju Severance Christian Hospital, Yonsei University Wonju College of Medicine, Wonju; ${ }^{2}$ Department of Rheumatology, Hanyang University Hospital for Rheumatic Diseases, Seoul; ${ }^{3}$ Division of Rheumatology, Department of Internal Medicine, Myongji Hospital, Hanyang University College of Medicine, Goyang; ${ }^{4}$ Department of Radiology, Kok Hospital, Seongnam; ${ }^{5}$ Department of Nuclear Medicine, Hanyang University Medical Center, Seoul; ${ }^{6}$ Department of Information Statistics, Yonsei University Mirae Campus, Wonju, Korea
\end{abstract}

Received: February 20, 2020 Revised : April 16, 2020 Accepted: April 27, 2020

\section{Correspondence to}

Tae-Hwan Kim, M.D.

Department of Rheumatology, Hanyang University Hospital for Rheumatic Diseases, 222 Wangsimni-ro, Seongdong-gu, Seoul 04763, Korea

Tel: +82-2-2290-9245

Fax: +82-2-2298-8231

E-mail: thkim@hanyang.ac.kr https://orcid.org/0000-00023542-2276
Backgrounds/Aims: This study was performed to reveal the usefulness of the trabecular bone score (TBS) in assessing bone strength in patients with ankylosing spondylitis (AS) in comparison with dual-energy X-ray absorptiometry (DXA) methods.

Methods: A total of 215 AS patients (75.8\% male) were enrolled from a single university hospital in Korea. Demographic and clinical information were assessed. Patients completed X-rays of the cervical and lumbar spine (L-spine), and spinal ankyloses were quantified using the modified Stoke AS Spine Score (mSASSS). Hip, anteroposterior and lateral L-spine bone mineral density (BMD) and TBS were assessed by DXA methods. Clinical characteristics and bone strength measurement results were compared between male and female AS patients. The accuracy of each bone strength evaluation method in predicting Fracture Risk Assessment Tool (FRAX) scores indicating moderate or higher fracture risk was compared by receiver operating characteristic curves in patients aged $\geq 40$ years. Correlations between each bone strength measurement method and mSASSS were examined.

Results: Male patients showed higher mSASSS and less prevalent peripheral joint involvement compared to female patients $(p<0.05)$. TBS, hip BMD, and L-spine lateral BMD showed comparably high areas under the curve (AUCs) for predicting FRAX-major osteoporotic fractures (MOF) $\geq 10 \%$ (AUC ranged 0.72 to 0.76 ). TBS negatively correlated with mSASSS in both male and female patients $(p<0.01)$. Conclusions: TBS could predict the risk of MOF and is not influenced by spinal osteoproliferation in AS patients, even in those with advanced spinal changes.

Keywords: Bone; Osteoporosis; Ankylosing spondylitis

\section{INTRODUCTION}

Ankylosing spondylitis (AS) is a chronic inflammatory disease of the axial skeleton. Cortical new bone formation leading to ankyloses of the spine and sacroiliac joints is a key characteristic of AS; however, AS is also associated with trabecular bone loss leading to osteopo- rosis $[1,2]$. Those opposite pathologic bone remodeling processes are an apparent paradox of this disease and take place in close proximity within the spine [1]. Indeed, patients with AS present with osteoporosis more frequently than the general population $[2,3]$. In addition, the characteristic osteoproliferation that increases the rigidity of the spine and bone loss in the vertebrae 
contributes to increased vulnerability of the spine in patients with AS. Previous studies have demonstrated that patients with AS have an increased risk of vertebral fracture (VF) that can be complicated by neurologic injuries $[4,5]$. Therefore, it is crucial to identify and selectively treat AS patients at risk for fractures.

Bone strength is determined by bone geometry, cortical thickness, porosity, trabecular bone morphology, and the intrinsic properties of bony tissue [6]. In clinical practice, bone strength is usually estimated indirectly from bone mineral density (BMD) determined using dual-energy X-ray absorptiometry (DXA) and the World Health Organization (WHO) definitions of osteopenia and osteoporosis [7]. Previous studies have found low BMD to be a good predictor of future fracture risk $[8,9]$. However, BMD corresponds to the ratio between the bone mineral content and the scanned bone area; it does not reflect bone architecture or quality. Moreover, it can be difficult to interpret lumbar spine BMD measured by DXA in the anteroposterior (AP) projection in advanced AS patients due to the presence of characteristic syndesmophytes [2,10-12]. Syndesmophytes develop in parallel to the progression of AS and thus mask the bone loss in AP spinal measurements of BMD [13]. Therefore, the European League Against Rheumatism recommended that patients with axial spondyloarthropathy (axSpA) who present with syndesmophytes on conventional lumbar spine radiography be assessed for osteoporosis by a hip DXA scan, supplemented by spine DXA in the lateral projection or possibly quantitative computed tomography (QCT) of the spine $[14,15]$. The trabecular bone score (TBS) is a recently developed tool for bone strength assessment that represents the textural parameter of pixel gray-level variations in lumbar spine DXA images [16]. Previous studies have shown that TBS could predict fractures independently of BMD and has additive value for discriminating patients with VF risk when combined with BMD [17-20]. In studies conducted in male AS patients [21] and patients with axSpA [11,12], TBS was not influenced by the characteristic syndesmophyte formation on the spine, unlike AP spine DXA. However, the data regarding the use of TBS in patients with AS was limited to a single study [21].

We aimed to reveal the usefulness of TBS for assessing bone strength in patients with AS in comparison with DXA methods (AP and lateral spine DXA and hip DXA) in a single-center AS cohort.

\section{METHODS}

\section{Study population}

Patients with AS who visited a single university hospital for periodic examinations between October 2016 and May 2017 were prospectively recruited to this study. All enrolled patients were diagnosed according to the modified New York criteria [22]. Patients who could not tolerate the supine position for BMD and patients with a history of lumbar surgery or surgery in both hips were excluded.

The study protocol was approved by the Institutional Review Board of Hanyang University Hospital, and informed consent was received from all participants (IRB no. HYUH 2016-08-006).

\section{Demographic and clinical characteristics}

Each participant completed questionnaires via interview regarding demographics, AS-related clinical information, and history of fracture. The history of fracture denoted whether the individual patient had ever experienced vertebral or non-VFs. Human leukocyte antigen (HLA) B27 positivity and medication history were checked by reviewing hospital medical records.

\section{Clinical and radiological parameters for the evalua- tion of AS}

Physical examinations for the Bath Ankylosing Spondylitis Metrology Index (BASMI), which measures spinal mobility in AS patients, were conducted by a single trained nurse [23]. Patients also completed questionnaires for the Bath Ankylosing Spondylitis Functional Index, a global visual analogue scale assessment (VAS-global), and the Bath Ankylosing Spondylitis Disease Activity Index (BASDAI) [24,25].

Lateral radiographs of the cervical and lumbar spine were acquired at the time of the BMD exam. Spinal changes related to AS were assessed according to the modified Stoke AS Spine Score (mSASSS) [26]. The mSASSS was assessed by a single expert radiologist at two different times for each patient, and the first measurement was used for evaluation. 


\section{Bone strength evaluation}

BMD of the hip (femoral neck and total hip) and lumbar spine in the AP (vertebrae $\mathrm{L}_{1}$ to $\mathrm{L}_{4}$ ) and lateral ( $\mathrm{L}_{2}$ to $\mathrm{L}_{3}$ ) projections was assessed by DXA (Discovery W, Hologic APEX software version 2.3.1, Hologic, Bedford, MA, USA) using a single device for the entire study. All BMD of the hip was estimated in the right hip. Patients were in the supine position for both the AP and lateral spine DXA scans to offer similar precision in both methods. Lumbar spine BMD was evaluated as the mean of the individual measurements for $\mathrm{L}_{1}$ to $\mathrm{L}_{4}$ and $\mathrm{L}_{2}$ to $\mathrm{L}_{3}$ in the $\mathrm{AP}$ projection and as the mean of the measurements for L2 and $\mathrm{L}_{3}$ in the lateral projection.

Lumbar spine AP DXA images were reanalyzed to calculate the TBS using TBS iNsight software version 2.0.0.1 (Med-Imaps, Bordeaux, France). Lumbar spine TBS was evaluated as the mean of the individual measurements for $\mathrm{L}_{1}$ to $\mathrm{L}_{4}$ and $\mathrm{L}_{2}$ to $\mathrm{L}_{3}$.

\section{Fracture risk assessment in AS patients aged 40 years or older}

Fracture Risk Assessment Tool for the \% 10-year probability of major osteoporotic fractures (FRAX-MOF) and hip fractures (FRAX-HF) were calculated by the FRAX tool (http://www.sheffield.ac.uk/FRAX) using known clinical risk factors. FRAX without BMD was used for the evaluation in this study to compare the usefulness of femur neck BMD with other bone strength measurement methods [27]. The FRAX tool was applied only to patients aged 40 years or older.

\section{Statistical analysis}

Data are expressed according to the properties of the variables. Demographic and clinical characteristics, BMD values, TBS, and the proportion of patients with abnormal bone strength were compared between male and female patients using Student's $t$ test, chi-square test, or Fisher's exact test, as appropriate. Abnormal bone strength was defined as T-score $<-1.0$ in patients aged 50 years or older in accordance with WHO definitions for osteopenia and osteoporosis [7]. Low TBS was defined as TBS $\leq 1.31$, following the results of recent studies $[17,21]$.

Fracture risk was categorized by the FRAX 10-year risk of fractures in patients aged $\geq 40$ years: (1) high fracture risk with FRAX-MOF $\geq 20 \%$ or FRAX-HF $\geq 3 \%$, and (2) moderate fracture risk with FRAX-MOF 10\% to $19 \%$ or FRAX-HF $>1 \%$ and $<3 \%$ [28]. The accuracy of BMD values and TBS in discriminating moderate or higher FRAX 10-year risk of fractures was assessed by determining the area under the receiver operating characteristic (ROC) curves. The relationships among the various BMD measurement results, TBS, and mSASSS were evaluated using Pearson's correlation coefficients. TBS and lumbar spine BMD measurement results were confined to mean values of the second and third lumbar spine measurements to evaluate those correlations. Statistical significance was defined as $p<0.05$. All statistical analyses were performed using IBM SPSS Statistics version 21.0 for Windows (IBM Corp., Armonk, NY, USA).

\section{RESULTS}

Among the 234 patients recruited for this study, 215 were included; eight patients did not complete the required physical exams, questionnaires, or BMD exam. One patient was eventually diagnosed with diffuse idiopathic skeletal hyperostosis rather than AS. Ten patients with a history of lumbar spine vertebroplasty or both hip replacement surgery were excluded from the study.

\section{Demographic and clinical characteristics}

The demographic and clinical characteristics of patients with AS included in this study are shown in Table 1. The overall mean \pm SD age of the patients was $49.9 \pm 10.4$ years, with a mean disease duration of $188.5 \pm 212.7$ months, and male patients were predominant (75.8\%). There were no significant differences in age or disease duration between male and female patients. Male patients showed higher body mass index and more prevalent smoking history and HLA B27 positivity ( $p<0.01$ ). Compared with female patients, male patients showed higher mSASSS and BASMI, reflecting more advanced spinal structural change $(p<0.01)$. In contrast, female patients showed more prevalent peripheral joint involvement and higher VAS-global and serum erythrocyte sedimentation rate levels. Overall, $25.6 \%$ of the study population had a history of fracture, and only nine patients $(4.2 \%)$ had a history of VF. Non-VF sites included wrist (5.1\% of the total population), ankle, finger, rib, tibia, pelvis, toe, shoulder, humerus, elbow, and knee (0.5\%), in order of frequency. 
Table 1. Demographic and clinical characteristics of patients with ankylosing spondylitis

\begin{tabular}{|c|c|c|c|c|}
\hline Characteristic & Total AS patients $(n=215)$ & Male $(n=163)$ & Female $(\mathrm{n}=52)$ & $p$ value \\
\hline Age, yr & $49.9 \pm 10.4$ & $49.6 \pm 10.8$ & $51.0 \pm 9.2$ & 0.34 \\
\hline Disease duration, mon & $188.5 \pm 212.7$ & $182.0 \pm 193.3$ & $208.9 \pm 265.4$ & 0.50 \\
\hline $\mathrm{BMI}, \mathrm{kg} / \mathrm{m}^{2}$ & $24 \cdot 5 \pm 3 \cdot 5$ & $25.0 \pm 3.5$ & $23.0 \pm 3.0$ & $<0.01$ \\
\hline Smoking, ever & $142(66)$ & $138(84 \cdot 7)$ & $4(7 \cdot 7)$ & $<0.01$ \\
\hline HLA B27 positivity $(\mathrm{n}=184)$ & $174(94 \cdot 6)$ & $132(97.8)(\mathrm{n}=135)$ & $42(85.7)(n=49)$ & $<0.01$ \\
\hline History of fracture & $55(25 \cdot 6)$ & $43(26.4)$ & $12(23.1)$ & 0.64 \\
\hline Vertebral fracture & $9(4.2)$ & $7(4 \cdot 3)$ & $2(3.8)$ & 1.00 \\
\hline Non-vertebral fracture & $47(21.9)$ & $37(22.7)$ & $10(19.2)$ & 0.60 \\
\hline Family history of AS & $55(25 \cdot 6)$ & $40(24.5)$ & $15(28.8)$ & 0.54 \\
\hline Peripheral joint involvement & $34(15.8)$ & $21(12.9)$ & $13(25 \cdot 0)$ & 0.04 \\
\hline VAS-global, 10-cm scale & $4.1 \pm 2.3$ & $3.9 \pm 2.2$ & $4.8 \pm 2.4$ & 0.01 \\
\hline BASDI & $3.4 \pm 1.9$ & $3.3 \pm 1.9$ & $3.8 \pm 2.1$ & 0.15 \\
\hline BASMI & $2.9 \pm 1.9$ & $3.2 \pm 1.8$ & $1.9 \pm 1.7$ & $<0.01$ \\
\hline mSASSS & $27.9 \pm 22.7$ & $32.5 \pm 23.1$ & $13.5 \pm 13.7$ & $<0.01$ \\
\hline $\mathrm{ESR}, \mathrm{mm} / \mathrm{hr}$ & $19.9 \pm 20.7$ & $18.2 \pm 20.9$ & $25 \cdot 3 \pm 19.0$ & 0.03 \\
\hline $\mathrm{CRP}, \mathrm{mg} / \mathrm{dL}$ & $0.4 \pm 1.0$ & $0.4 \pm 1.0$ & $0.3 \pm 0.8$ & 0.27 \\
\hline C-telopeptide, $\mu \mathrm{g} / \mathrm{mL}(\mathrm{n}=189)$ & $0.3 \pm 0.2$ & $0.3 \pm 0.1(n=145)$ & $0.3 \pm 0.2(\mathrm{n}=44)$ & 0.24 \\
\hline Osteocalcin, $\mathrm{ng} / \mathrm{mL}(\mathrm{n}=2 \mathrm{O} 3)$ & $18.6 \pm 6.5$ & $18.1 \pm 5 \cdot 4(\mathrm{n}=153)$ & $20.1 \pm 8.9(n=50)$ & 0.14 \\
\hline \multicolumn{5}{|l|}{ History of medication use } \\
\hline NSAIDs & $187(87.0)$ & $142(87.1)$ & $45(86.5)$ & 0.91 \\
\hline DMARDs & $41(19.1)$ & $28(17.2)$ & $13(25 \cdot 0)$ & 0.21 \\
\hline Methotrexate & $18(8.4)$ & $12(7 \cdot 4)$ & $6(11.5)$ & 0.39 \\
\hline Sulfasalazine & $31(14 \cdot 4)$ & $20(12.3)$ & $11(21.2)$ & 0.11 \\
\hline Glucocorticoids $^{\mathrm{a}}$ & $80(37.2)$ & $65(39.9)$ & $15(28.8)$ & 0.15 \\
\hline Biologics & $124(57 \cdot 7)$ & $98(60.1)$ & $26(50.0)$ & 0.20 \\
\hline Anti-osteoporotic agents & $9(4.2)$ & $5(3.1)$ & $4(7 \cdot 7)$ & 0.23 \\
\hline
\end{tabular}

Values are presented as mean $\pm \mathrm{SD}$ or number (\%).

AS, ankylosing spondylitis; BMI, body mass index; HLA, human leukocyte antigen; VAS, visual analogue scale; BASDAI, Bath Ankylosing Spondylitis Disease Activity Index; BASMI, Bath Ankylosing Spondylitis Metrology Index; mSASSS, modified Stoke AS Spine Score; ESR, erythrocyte sedimentation rate; CRP, C-reactive protein; C-telopeptide, C-terminal telopeptide; NSAID, nonsteroidal anti-inflammatory drug; DMARD, disease modifying antirheumatic drug.

${ }^{\mathrm{a}}$ History of glucocorticoid use equivalent to $5 \mathrm{mg}$ or more of prednisolone for more than 3 months.

There were no significant differences between male and female patients in the prevalence of vertebral or non-VFs or the use of each medication.

\section{Comparison of bone strength measurement results between male and female AS patients}

The results of each bone strength measurement and the proportions of patients with abnormal bone strength are compared in Table 2. Male patients showed higher bone strength regardless of the measurement method. However, the difference was not statistically significant for TBS. Lumbar spine BMD of L2 to L3 was higher in the AP scan than in the lateral DXA scan, probably because the two scan methods include different parts of vertebrae and related structures $\left(1.04 \pm 0.21 \mathrm{~g} / \mathrm{cm}^{2}\right.$ in AP scan vs. $0.62 \pm 0.17 \mathrm{~g} / \mathrm{cm}^{2}$ in lateral scan). In patients aged 50 years or older, the proportion of female patients with abnormal bone strength was higher than in male pa- 
Table 2. Comparison of bone strength measurements in patients with ankylosing spondylitis

\begin{tabular}{|c|c|c|c|c|}
\hline Parameter & Total AS patients $(n=215)$ & Male $(n=163)$ & Female $(n=52)$ & $p$ value \\
\hline \multicolumn{5}{|l|}{ Hip BMD, g/cm ${ }^{2}$} \\
\hline Femur neck & $0.74 \pm 0.13$ & $0.76 \pm 0.13$ & $0.68 \pm 0.09$ & $<0.01$ \\
\hline Total & $0.87 \pm 0.13$ & $0.89 \pm 0.13$ & $0.79 \pm 0.10$ & $<0.01$ \\
\hline Lumbar spine $\mathrm{BMD}, \mathrm{g} / \mathrm{cm}^{2}$ & $1.04 \pm 0.21$ & $1.07 \pm 0.21$ & $0.92 \pm 0.14$ & $<0.01$ \\
\hline \multicolumn{5}{|l|}{ Lumbar spine BMD (L2-L3), g/cm² } \\
\hline Anteroposterior & $1.04 \pm 0.21$ & $1.07 \pm 0.22$ & $0.92 \pm 0.14$ & $<0.01$ \\
\hline Lateral & $0.62 \pm 0.17$ & $0.64 \pm 0.18$ & $0.55 \pm 0.12$ & $<0.01$ \\
\hline TBS & $1.39 \pm 0.10$ & $1.37 \pm 0.10$ & $1.35 \pm 0.08$ & 0.19 \\
\hline TBS (L2-L3) & $1.39 \pm 0.10$ & $1.40 \pm 0.11$ & $1.36 \pm 0.09$ & 0.06 \\
\hline Patients with abnormal bone strength ${ }^{a}$ & 114 & 83 & 31 & \\
\hline \multicolumn{5}{|l|}{ Osteoporosis or osteopenia ${ }^{\mathrm{b}}$} \\
\hline Femur neck BMD & $61(53 \cdot 5)$ & $38(45 \cdot 8)$ & $23(74.2)$ & $<0.01$ \\
\hline Total hip BMD & $30(26.3)$ & $17(20.5)$ & $13(41.9)$ & 0.02 \\
\hline Lumbar spine BMD & $32(28.1)$ & $15(18.1)$ & $17(54.8)$ & $<0.01$ \\
\hline Low TBS of lumbar spine $(\leq 1.31)$ & $28(24.6)$ & $14(16.9)$ & $14(45 \cdot 2)$ & $<0.01$ \\
\hline
\end{tabular}

Values are presented as mean $\pm \mathrm{SD}$, number only, or number $(\%)$.

AS, ankylosing spondylitis; BMD, bone mineral density; L2-L3, mean of L2 and L3; TBS, trabecular bone score.

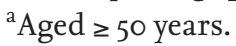

${ }^{\mathrm{b}} \mathrm{T}$ score $\leq-1.0$.

Table 3. ROC AUC values to discriminate moderate or higher 10 year probability of fracture in patients aged 40 years or older

\begin{tabular}{|c|c|c|c|c|c|c|}
\hline \multirow{2}{*}{ Variable } & \multirow{2}{*}{ Number } & \multicolumn{2}{|c|}{ Hip BMD } & \multicolumn{2}{|c|}{ Lumbar spine BMD (L2-L3) } & \multirow{2}{*}{ TBS (L2-L3) } \\
\hline & & Total & Femoral neck & Anteroposterior & Lateral & \\
\hline $\begin{array}{l}\text { Ten-year probability of fracture } \\
\text { by FRAX without BMD }\end{array}$ & 173 & & & & & \\
\hline FRAX-MOF $\geq 20 \%$ & 2 & NA & NA & NA & NA & NA \\
\hline FRAX-MOF $\geq 10 \%$ & 11 & 0.76 & 0.73 & 0.61 & 0.72 & 0.73 \\
\hline FRAX-HF $\geq 3 \%$ & 10 & 0.73 & 0.75 & 0.61 & 0.73 & 0.64 \\
\hline FRAX-HF $\geq 1 \%$ & 54 & 0.66 & 0.68 & 0.56 & 0.62 & 0.53 \\
\hline
\end{tabular}

ROC, receiver operating characteristics; AUC, area under the curve; BMD, bone mineral density; FRAX, Fracture Risk Assessment Tool; MOF, major osteoporotic fracture; NA, not applicable; HF, hip fracture.

tients regardless of the evaluation method.

\section{Accuracy of bone strength evaluation methods to detect FRAX scores indicating moderate or higher fracture risk in AS patients aged 40 years or older}

As shown in Table 3, the ROC curve for discriminating FRAX-MOF scores indicating a high fracture risk was not drawn properly because only two patients showed a
FRAX-MOF score of high fracture risk. All bone strength measurements showed comparably high AUCs to detect a FRAX-MOF score indicating a moderate or higher fracture risk (FRAX-MOF $\geq 10 \%$; AUCs ranged 0.72 to 0.76), except lumbar spine AP BMD. However, the AUCs for discriminating a FRAX-HF indicating a moderate or higher fracture risk were lower for the lumbar spine AP BMD and TBS than for the other methods tested. 
Table 4. Correlations between bone strength measurements and modified Stoke Ankylosing Spondylitis Spine Score

\begin{tabular}{|c|c|c|c|c|c|}
\hline \multirow{2}{*}{ Patient } & \multicolumn{2}{|c|}{ Hip BMD } & \multicolumn{2}{|c|}{ Lumbar spine BMD (L2-L3) } & \multirow{2}{*}{ TBS (L2-L3) } \\
\hline & Total & Femoral neck & Anteroposterior & Lateral & \\
\hline Total $(n=215)$ & $0.200^{\mathrm{a}}$ & $0.187^{\mathrm{a}}$ & $0.402^{\mathrm{a}}$ & 0.009 & $-0.182^{\mathrm{a}}$ \\
\hline Male $(n=163)$ & 0.149 & 0.154 & $0.393^{\mathrm{a}}$ & -0.056 & $-0.240^{\mathrm{a}}$ \\
\hline Female $(\mathrm{n}=52)$ & $-0.341^{b}$ & $-0.316^{b}$ & -0.219 & $-0.287^{b}$ & $-0.296^{b}$ \\
\hline
\end{tabular}

By Pearson's correlation coefficients.

$\mathrm{BMD}$, bone mineral density; L2-L3, mean of L2 and L3; TBS, trabecular bone score.

${ }^{\mathrm{a}}$ Correlation is significant at the 0.01 level.

${ }^{\mathrm{b}}$ Correlation is significant at the 0.05 level.

\section{Correlations between bone strength measurement methods and mSASSS}

The correlations between all the bone strength measurement methods and mSASSS are presented in Table 4. The most notable finding is that only TBS negatively correlated with mSASSS in both male and female patients with AS $(r=-0.240$ and $r=-0.296$, respectively; $p$ $<0.01$ ). TBS also negatively correlated with mSASSS in the total study population. In contrast, lumbar spine AP BMD showed a positive correlation with mSASSS in male patients and all patients. There was no correlation between lumbar spine AP BMD and mSASSS in female patients. However, hip BMD and lumbar spine lateral BMD showed negative correlations with mSASSS only in female patients $(p<0.05)$.

\section{DISCUSSION}

Our study population reflects the characteristic clinical differences between male and female patients with AS in Korea. In addition, TBS showed comparable predictability for detecting FRAX-MOF score of moderate or higher fracture risk compared to hip BMD or lumbar lateral $\mathrm{BMD}$ in the analyses of ROC curves in AS patients aged 40 years or older. This indicates that a low TBS could be associated with an increased risk of MOF that includes VF in patients with AS. The most distinctive finding of this study is that only TBS negatively correlated with mSASSS in both male and female AS patients. In other words, TBS was the only bone strength measurement that was unaffected by the characteristic spinal osteoproliferation of AS, even in male patients with advanced spi- nal changes. These key findings attest to the usefulness of TBS in assessing bone strength in patients with AS.

Many previous studies have shown that clinical characteristics could differ between male and female patients. Male AS patients generally show more prevalent HLA B27 positivity and advanced spinal radiographic change. In contrast, female patients present with more prevalent peripheral joint involvement and higher symptomatic burden of AS [29]. Our study showed similar characteristic differences between male and female AS patients, even though age and disease duration did not differ between the groups (Table 1). However, the similar BASDAI and C-reactive protein (CRP) found in female patients in this study differed from the findings of previous studies, which mainly included white ethnicity populations. BASDAI and CRP were higher in female patients in those previous studies [29]. As shown in Fig. 1, the distribution of patients according to mSASSS is distinctively right skewed, and at all spine levels, the mean grades of mSASSS were higher in male patients than in female patients. Thus, sex seems to play a very important role in AS and should always be regarded as an important confounding factor when investigating patients with AS. However, no previous study has separately evaluated correlations between bone strength measurement methods and the degree of spinal radiographic change in male and female AS patients [21].

The prevalence of VF history in this study was $4.2 \%$, which was lower than the prevalence of $13.6 \%$ in the general population of a rural Korean community [30]. In a Swedish cohort of 204 AS patients by Klingberg et al. [15], only three patients (1\%) reported previous VF. However, 24 patients (11.8\%) were found to have VF after the sys- 

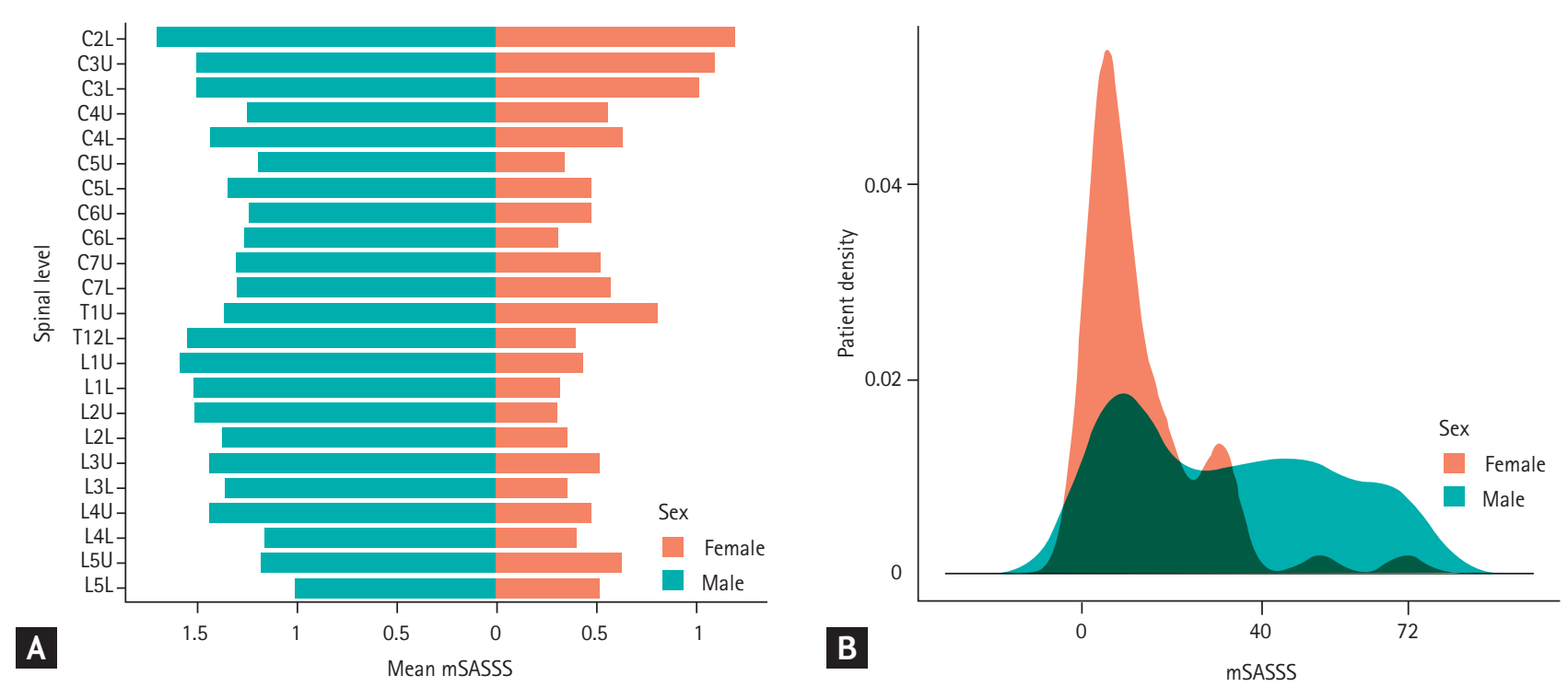

Figure 1. Comparison of modified Stoke Ankylosing Spondylitis Spine Score (mSASSS) between male and female patients with ankylosing spondylitis. (A) Mean mSASSS for each spinal level. (B) Distribution of patient density according to the mSASSS. C, cervical spine; T, thoracic spine; L, lumbar spine; the upper and lower anterior corners of the spines are denoted by $\mathrm{U}$ and L, respectively.

temic radiographic evaluation. The presence of VF was also determined by systemic radiographic evaluation in the cohort of a Korean rural community [30]. The ankylosed spine of AS patients is prone to fracture even after minor trauma, and spinal fractures are asymptomatic in some patients, leading to delay in diagnosis. Furthermore, X-ray of the spine in patients with AS may be difficult to interpret owing to the preexisting spinal structural change of AS [5]. Klingberg et al. [15] also showed that the prevalence of VF varied among studies from $9 \%$ to $42 \%$ depending on different definitions of VF, making a straightforward comparison between studies impossible. The prevalence of non-VF in our study was higher than in the Swedish cohort result (21.9\% vs. 9\%) [15]. However, both studies have limitation for comparison of fracture prevalence as traumatic causes were not distinguished.

In general, males tend to show higher BMD compared to females both at the lumbar spine and hip [31]. Measurement of BMD in this study was also higher in male patients at every examined site (Table 2). But, TBS is a pure bone texture index that measures different aspects of bone strength independent of BMD [16,32]. No significant difference in TBS between the sexes was noted in this study, like the results of a previous study conducted in the general population [32]. Indeed, there was no previ- ous study to compare with our results regarding the TBS differences between male and female AS patients. However, patients with low TBS $(\leq 1.31)$ were more prevalent in female in patients aged 50 years or older, as shown in Table 2. Female AS patients aged 50 years or older could be at higher risk of fracture compared to male patients of same age group in aspects of bone density and trabecular bone quality. However, predicting future fracture risk is a complicated task because many clinical factors other than BMD can contribute to it. Those clinical factors include age, sex, prior fragility fractures, parental history of hip fracture, smoking history, alcohol consumption, long-term glucocorticoid use, and certain comorbidities such as rheumatoid arthritis [33]. FRAX is an algorithm developed by WHO that calculates 10-year fracture probability using those clinical factors. Our results show that hip BMD, lumbar lateral BMD, and TBS predict a moderate or higher risk of FRAX-MOF comparably well in patients aged 40 years or older. FRAX-MOF reflects the probability of fracture in the hip, clinical spine, wrist, or humerus [33]. TBS was not inferior in predicting MOF compared to hip DXA scan in this group of AS patients. In a previous study of patients with axSpA, TBS was lower in patients with VF than in those without VF, and TBS showed better discriminatory value than total hip BMD in the prediction of VF [34]. Our result, taken together 
with previous reports, support the idea that TBS could predict future VF in patients with AS. However, FRAX has the limitation of not considering many causes of secondary osteoporosis [33]. According to a study on the clinical risk factors for VF in AS patients, the presence of VF was significantly associated with higher mSASSS [35]. This result suggests that the risk of VF not only increases with the presence of the disease itself, but also with more advanced spinal structural change of AS. Therefore, we need to improve the clinical tools available to predict the future risk of VF in AS patients. The presence of AS and the severity of its spinal change could be good candidate clinical risk factors to consider when predicting the risk of VF in patients with AS [4,5].

Our study showed some contradictory results compared with two previous studies regarding the correlations between bone strength measurement methods and mSASSS. Femoral neck, total hip, and lumbar lateral BMD negatively correlated with mSASSS in the Swedish cohort of 204 AS patients [2]. In a Korean cohort of 100 male AS patients aged 50 years or younger by Kang et al. [21], femoral neck and total hip BMD also negatively correlated with SASSS, another radiographic scoring system that measures only lumbar spinal changes in AS patients. However, in our study, lumbar lateral BMD and hip BMD correlated negatively with mSASSS only in female patients, not in male patients. The Swedish group reported that their five-year follow-up data showed unexpected increase in lumbar lateral and total hip BMD but decrease in femoral neck BMD when mSASSS increased. Because the increase in lumbar AP and lateral BMD was significant only for male patients, who had higher mSASSS than female patients overall, they suspected that the lumbar lateral DXA scan might also have been influenced by osteoproliferation of spine [36]. Male patients in our study included patients with much higher mSASSS than the patients in the Swedish cohort (mSASSS of $32.5 \pm 23.1$ in this cohort vs. $16.6 \pm 20.9$ in the Swedish cohort) and the patients in the Korean cohort conducted by Kang et al. [21] (SASSS of $13.71 \pm 13.26$ in this cohort vs. 9.5 \pm 16.0 in the young male Korean AS cohort). Therefore, we also assume that advanced spinal structural changes could have affected some part of the lumbar spine and increased the lumbar lateral BMD. The increase in total hip BMD after 5 years of follow up in the Swedish cohort was also significant only in male patients $(p<0.001)$. Zhao et al. [37] in- vestigated the factors associated with severe radiographic hip involvement in an AS cohort and identified spinal involvement as an associated clinical factor. Accompanying hip cortical changes, such as osteophyte formation, could have increased both total hip and femur neck BMD in male patients with advanced spinal disease in our cohort. Future studies that measure radiographic hip change or perform a QCT evaluation of both hip and lumbar spine could help to clarify these correlations.

One of the most notable findings in this study is that only TBS correlated negatively with mSASSS in both male and female AS patients. Though TBS also correlated negatively with SASSS in the cohort of young male AS patients by Kang et al. [21], our finding signifies that TBS is not influenced by the spinal osteoproliferation characteristic of AS, even in male patients with advanced spinal changes and female AS patients. The strengths of TBS include easy accessibility and low cost because it is calculated using lumbar AP DXA scan images [16]. In addition, compared with lumbar lateral DXA scans or QCT, TBS has fewer problems with poor precision or radiation hazards, respectively $[2,38]$. Because only a single study previously used TBS for patients with AS [21], more studies on this topic are needed, and TBS should be considered as an additional recommended tool for evaluating osteoporosis in patients with AS.

Our study has several limitations. First, we investigated the prevalence of fracture primarily by relying on patient history, without systemic X-ray evaluations for VF. However, we reviewed all the medical records of spinal X-ray interpretation to minimize this weakness. In addition, three patients with history of lumbar surgery were excluded in this study to facilitate accurate bone strength evaluation. Therefore, our result could underestimate the true prevalence of VF to some extent. Second, we did not examine the menopausal status in female patients, which has a significant impact on female bone health [39]. Park et al. [40] reported the mean \pm SD age of natural menopause in Korea as $49.3 \pm 0.07$ years through the evaluation of 12,761 females using the Korea National Health and Nutrition Examination Survey data. Therefore, in the evaluation of patients with abnormal bone strength (Table 2), we limited female patients to aged 50 years or older as most of them are likely to be in postmenopausal or late perimenopausal period, when bone loss is accelerated owing to the hor- 
monal change [39]. Third, because we did not perform QCT evaluations, the exact degree of cortical or trabecular change of the spine or hip was not evaluated. Lastly, mSASSS was measured by a single investigator at two different times. However, the intraobserver reliability between the two measurements was excellent (intraclass correlation coefficients, 1.00; $p<0.001$ ).

In summary, among the bone strength evaluation methods examined in this study, only TBS was not influenced by the characteristic spinal osteoproliferation of AS in both male and female patients. In addition, TBS was not inferior in predicting the risk of MOF compared to hip BMD or lumbar spine lateral BMD. Lumbar spine AP BMD was inferior in predicting the risk of MOF compared to other methods. Our results indicate that TBS is a useful tool in assessing bone strength in patients with AS.

\section{KEY MESSAGE}

1. Trabecular bone score (TBS) can reflect the risk of major osteoporotic fracture in patients with ankylosing spondylitis (AS).

2. Both lumbar lateral and hip bone mineral density (BMD) results could be distorted in patients with AS.

3. Lumbar anteroposterior BMD is inferior in assessing bone strength in AS patients.

4. TBS is unaffected by spinal osteoproliferation both in male and female AS patients.

5. TBS is a useful tool in assessing bone strength in patients with AS.

\section{Conflict of interest}

No potential conflict of interest relevant to this article was reported.

\section{REFERENCES}

1. Carter S, Lories RJ. Osteoporosis: a paradox in ankylosing spondylitis. Curr Osteoporos Rep 2011;9:112-115.

2. Klingberg E, Lorentzon M, Mellstrom D, et al. Osteoporosis in ankylosing spondylitis: prevalence, risk factors and methods of assessment. Arthritis Res Ther 2012;14:R108.
3. Kang KY, Kwok SK, Ju JH, Hong YS, Park SH. Assessment of fracture risk in patients with axial spondyloarthritis: a case-control study using the fifth Korean National Health and Nutrition Examination Survey (KNHANES V). Scand J Rheumatol 2016;45:23-31.

4. Vosse D, Landewe R, van der Heijde D, van der Linden S, van Staa TP, Geusens P. Ankylosing spondylitis and the risk of fracture: results from a large primary care-based nested case-control study. Ann Rheum Dis 2009;68:1839-1842.

5. Westerveld LA, Verlaan JJ, Oner FC. Spinal fractures in patients with ankylosing spinal disorders: a systematic review of the literature on treatment, neurological status and complications. Eur Spine J 2009;18:145-156.

6. Ammann P, Rizzoli R. Bone strength and its determinants. Osteoporos Int 2003;14 Suppl 3:S13-S18.

7. Assessment of fracture risk and its application to screening for postmenopausal osteoporosis. Report of a WHO Study Group. World Health Organ Tech Rep Ser 1994;843:1-129.

8. Johnell O, Kanis JA, Oden A, et al. Predictive value of BMD for hip and other fractures. J Bone Miner Res 2005;20:1185-1194.

9. Marshall D, Johnell O, Wedel H. Meta-analysis of how well measures of bone mineral density predict occurrence of osteoporotic fractures. BMJ 1996;312:1254-1259.

10. Kaya A, Ozgocmen S, Kamanli A, Ardicoglu O. Bone loss in ankylosing spondylitis: does syndesmophyte formation have an influence on bone density changes? Med Princ Pract 2009;18:470-476.

11. Wildberger L, Boyadzhieva V, Hans D, Stoilov N, Rashkov $\mathrm{R}$, Aubry-Rozier B. Impact of lumbar syndesmophyte on bone health as assessed by bone density (BMD) and bone texture (TBS) in men with axial spondyloarthritis. Joint Bone Spine 2017;84:463-466.

12. Kang KY, Goo HY, Park SH, Hong YS. Trabecular bone score as an assessment tool to identify the risk of osteoporosis in axial spondyloarthritis: a case-control study. Rheumatology (Oxford) 2018;57:587.

13. Ulu MA, Cevik R, Dilek B. Comparison of PA spine, lateral spine, and femoral BMD measurements to determine bone loss in ankylosing spondylitis. Rheumatol Int 2013;33:1705-1711.

14. Mandl P, Navarro-Compan V, Terslev L, et al. EULAR recommendations for the use of imaging in the diagnosis and management of spondyloarthritis in clinical practice. Ann Rheum Dis 2015;74:1327-1339. 
15. Klingberg E, Geijer M, Gothlin J, et al. Vertebral fractures in ankylosing spondylitis are associated with lower bone mineral density in both central and peripheral skeleton. J Rheumatol 2012;39:1987-1995.

16. Harvey NC, Gluer CC, Binkley N, et al. Trabecular bone score (TBS) as a new complementary approach for osteoporosis evaluation in clinical practice. Bone 2015;78:216-224.

17. McCloskey EV, Oden A, Harvey NC, et al. A meta-analysis of trabecular bone score in fracture risk prediction and its relationship to FRAX. J Bone Miner Res 2016;31:940-948.

18. Kim D, Cho SK, Kim JY, Choi YY, Sung YK. Association between trabecular bone score and risk factors for fractures in Korean female patients with rheumatoid arthritis. Mod Rheumatol 2016;26:540-545.

19. Hans D, Goertzen AL, Krieg MA, Leslie WD. Bone microarchitecture assessed by TBS predicts osteoporotic fractures independent of bone density: the Manitoba study. J Bone Miner Res 2011;26:2762-2769.

20. Briot K, Paternotte S, Kolta S, et al. Added value of trabecular bone score to bone mineral density for prediction of osteoporotic fractures in postmenopausal women: the OPUS study. Bone 2013;57:232-236.

21. Kang KY, Chung MK, Kim HN, Hong YS, Ju JH, Park SH. Severity of sacroiliitis and erythrocyte sedimentation rate are associated with a low trabecular bone score in young male patients with ankylosing spondylitis. J Rheumatol 2018;45:349-356.

22. van der Linden S, Valkenburg HA, Cats A. Evaluation of diagnostic criteria for ankylosing spondylitis. A proposal for modification of the New York criteria. Arthritis Rheum 1984;27:361-368.

23. Jenkinson TR, Mallorie PA, Whitelock HC, Kennedy LG, Garrett SL, Calin A. Defining spinal mobility in ankylosing spondylitis (AS). The Bath AS Metrology Index. J Rheumatol 1994;21:1694-1698.

24. Calin A, Garrett S, Whitelock H, et al. A new approach to defining functional ability in ankylosing spondylitis: the development of the Bath Ankylosing Spondylitis Functional Index. J Rheumatol 1994;21:2281-2285.

25. Garrett S, Jenkinson T, Kennedy LG, Whitelock H, Gaisford P, Calin A. A new approach to defining disease status in ankylosing spondylitis: the Bath Ankylosing Spondylitis Disease Activity Index. J Rheumatol 1994;21:2286-2291.

26. Creemers MC, Franssen MJ, van't Hof MA, Gribnau FW, van de Putte LB, van Riel PL. Assessment of outcome in ankylosing spondylitis: an extended radiographic scoring system. Ann Rheum Dis 2005;64:127-129.

27. Gadam RK, Schlauch K, Izuora KE. Frax prediction without BMD for assessment of osteoporotic fracture risk. Endocr Pract 2013;19:780-784.

28. Buckley L, Guyatt G, Fink HA, et al. 2017 American College of Rheumatology guideline for the prevention and treatment of glucocorticoid-induced osteoporosis. Arthritis Rheumatol 2017;69:1521-1537.

29. van der Horst-Bruinsma IE, Zack DJ, Szumski A, Koenig AS. Female patients with ankylosing spondylitis: analysis of the impact of gender across treatment studies. Ann Rheum Dis 2013;72:1221-1224.

30. Shin CS, Kim MJ, Shim SM, et al. The prevalence and risk factors of vertebral fractures in Korea. J Bone Miner Metab 2012;30:183-192.

31. Alswat KA. Gender disparities in osteoporosis. J Clin Med Res 2017;9:382-387.

32. Bazzocchi A, Ponti F, Diano D, et al. Trabecular bone score in healthy ageing. Br J Radiol 2015;88:20140865.

33. Kanis JA, Hans D, Cooper C, et al. Interpretation and use of FRAX in clinical practice. Osteoporos Int 2011;22:2395-2411.

34. Kang KY, Kim IJ, Park SH, Hong YS. Associations between trabecular bone score and vertebral fractures in patients with axial spondyloarthritis. Rheumatology (Oxford) 2018;57:1033-1040.

35. Maas F, Spoorenberg A, van der Slik BPG, et al. Clinical risk factors for the presence and development of vertebral fractures in patients with ankylosing spondylitis. Arthritis Care Res (Hoboken) 2017;69:694-702.

36. Deminger A, Klingberg E, Lorentzon M, et al. Which measuring site in ankylosing spondylitis is best to detect bone loss and what predicts the decline: results from a 5-year prospective study. Arthritis Res Ther 2017;19:273.

37. Zhao J, Zheng W, Zhang C, Li J, Liu D, Xu W. Radiographic hip involvement in ankylosing spondylitis: factors associated with severe hip diseases. J Rheumatol 2015;42:106-110.

38. Kopperdahl DL, Aspelund T, Hoffmann PF, et al. Assessment of incident spine and hip fractures in women and men using finite element analysis of CT scans. J Bone Miner Res 2014;29:570-580.

39. Lo JC, Burnett-Bowie SA, Finkelstein JS. Bone and the perimenopause. Obstet Gynecol Clin North Am 2011;38:503-517.

40. Park CY, Lim JY, Park HY. Age at natural menopause in Koreans: secular trends and influences thereon. Menopause 2018;25:423-429. 